\title{
Desarrollo tecnológico de un sistema espectral portátil con luz blanca para la captación de huellas en hojas de plantas florícolas
}

\author{
Fredy Paulino Chaglla Sisalema
} Escuela Politécnica Nacional. Ingeniería Eléctrica y Electrónica fredy.chaglla@epn.edu.ec

Paolo Andrés Pancho Ramírez Escuela Politécnica Nacional. Ingeniería Eléctrica y Electrónica paolo.pancho@epn.edu.ec

Fausto Eduardo Ávalos Cascante Escuela Politécnica Nacional eduardo.avalos@epn.edu.ec

Rolando Sáenz

Escuela Politécnica Nacional rolando.saez@epn.edu.ec

Recibido: 01 de septiembre / Aprobado: 30 de noviembre 2017

\section{Resumen}

En el presente artículo se expone el diseño e implementación de un equipo prototipo, utilizando luz blanca para la captación de huellas en hojas de plantas florícolas. El equipo prototipo consta de tres principales módulos constitutivos que son el sistema óptico, el sistema electrónico y el software de visualización.

Palabras clave: sistema espectral, luz blanca, plantas florícolas, sistema óptico, sistema electrónico, software de visualización. 


\section{Abstract}

In the present article the design and implementation of a prototype equipment is introduced to the readers. The prototype uses white light for the capture of traces in leaves of floricultural plants. The prototype equipment consists of three main constitutive modules that are the optical system, the electronic system and the visualization software.

Keywords: spectral system, white light, flower plants, optical system, electronic system, visualization software. 


\section{Introducción}

$\mathrm{E}$

n el presente documento se presenta el diseño e implementación de un equipo prototipo, utilizando luz blanca para la captación de huellas en hojas de plantas florícolas. El equipo prototipo consta de tres módulos constitutivos que son: el sistema óptico, el sistema electrónico y el software de visualización

\section{Diseño e implementación del sistema óptico}

En esta sección, se detalla el diseño e implementación del sistema óptico del equipo, que está conformado por la fuente de luz, el detector y la punta de toma de muestras.

\section{a. Fuente de luz}

El detector trabaja en la región visible del espectro electromagnético, por lo que se utiliza una lámpara halógena de Ocean Optics.

Esta lámpara se escogió debido a que se ajusta al rango del espectro en donde se trabaja para la obtención de las gráficas, este rango va desde los $200 \mathrm{~nm}$ a $850 \mathrm{~nm}$. Además, esta lámpara está focalizada de fábrica [1], permitiendo que la luz producida no se disperse, sino que se concentre en un punto. La luz generada por la fuente de luz blanca es enviada hacia las muestras, con las que interactúa produciendo una respuesta que es procesada por un detector y convertida en datos que posteriormente se transforman en gráficas como se muestra en la figura 1.

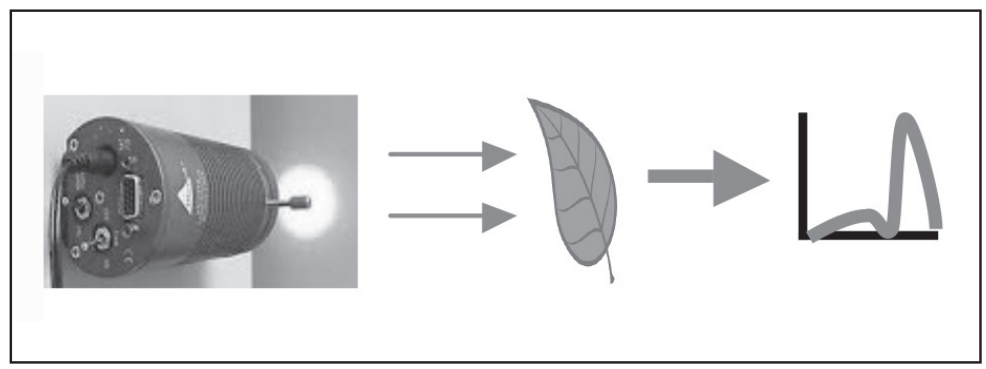

Figura 1. Representación gráfica de la función de la fuente de luz.

La fuente de luz está conformada por los siguientes elementos:

- Disipador

- Lente colimador

- Fibra óptica

El disipador está construido de aluminio, debido a su propiedad conductora que ayuda a transferir el calor de la fuente al exterior y a su peso, que es menor en comparación con el cobre que también es un buen disipador, pero mucho más pesado. 
El lente colimador es el encargado de enlazar la fibra óptica y el detector con la fuente de luz [2], debido a sus características técnicas. El detector y la fibra óptica cuentan con una rosca de estándar de 3/8 de pulgada (inch) con la que el lente colimador se enrosca perfectamente a estos, además, sirve para realizar el enfoque adecuado [3], consta de una rosca móvil que permite ajustar la distancia de acople y así ubicar el plano focal a una distancia específica.

La fibra óptica es la encargada de transmitir la luz hacia las muestras y hacia el detector. Consta de un revestimiento, que permite su uso en campo, además protege a la fibra de movimientos mecánicos, también consta de dos canales que permite la interacción de la fuente de luz con las muestras y el detector USB-4000. Las características de la fibra se acoplan a las necesidades del equipo [4], que son el rango de longitud de onda de trabajo y la rosca para el acople con el detector y la fuente de luz.

\section{b. Punta de toma de muestras}

En el equipo prototipo la punta de toma de muestras es la encargada de formar una cápsula cerrada, evitando que el ruido del ambiente interfiera con la toma de datos. El ruido para el equipo es la luz ambiental, por tal razón la punta de toma de muestras dispone de dos caras las cuales se unen paralelamente. Al introducir la hoja entre las paredes de la punta de toma de muestras estas se sellan evitando el ruido del ambiente.

La punta de toma de muestras está construida de manera que minimice el esfuerzo de la mano en el momento de toma de muestras, tiene la forma de una pinza, construida de dos partes principales, la parte superior porta la fibra óptica la cual es ajustada por medio de un tornillo de sujeción y la parte inferior de la pinza dispone de una cara paralela a la superior. Se la puede observar en la figura 2.

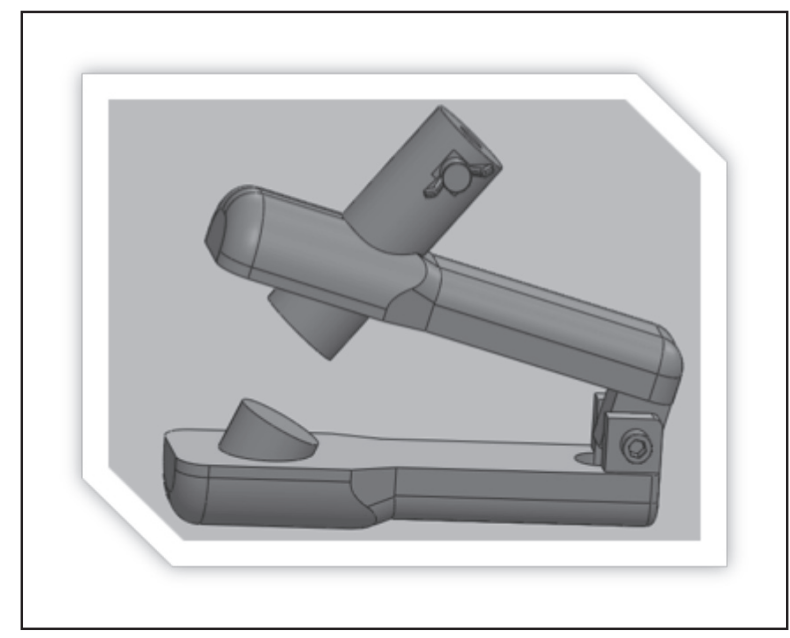

Figura 2. Punta de toma de muestras. 
c. Detector

El espectrómetro USB4000 UV-VIS-ES de Ocean Optics es un equipo que contiene un sistema óptico, combinado con la electrónica, se distingue de las demás versiones por su alta sensibilidad, posee un detector de 3648 elementos con obturador, trabaja desde 200 a $850 \mathrm{~nm}$, se conecta a un computador mediante un puerto USB 2.0 o 1.1, la alimentación la extrae del puerto USB.

El detector utilizado para el espectrómetro USB-4000 UV-VIS-ES es un dispositivo de transferencia de carga CCD (Charge Coupled Device) el cual consta de un arreglo lineal de sensores conformados por fotodetectores, [5] este tipo de sensor se encuentra comúnmente en equipos de fotografía, por su sensibilidad a la luz.

Para el detector usado en el equipo prototipo, la transferencia de carga, el reinicio y la iniciación de la lectura empiezan con el reloj en $1 \mathrm{~L}$ (uno lógico), en el tiempo de integración. La función de reinicio recarga los fotodetectores y permite la integración continua de la energía luminosa durante el tiempo de integración, mientras se leen los datos a través de registros de desplazamiento en serie.

Al final de un período de integración, el proceso se repite. El CCD es un dispositivo de agotamiento y la señal eléctrica de salida es inversamente proporcional a los fotones de entrada, mostrada en la figura 3.

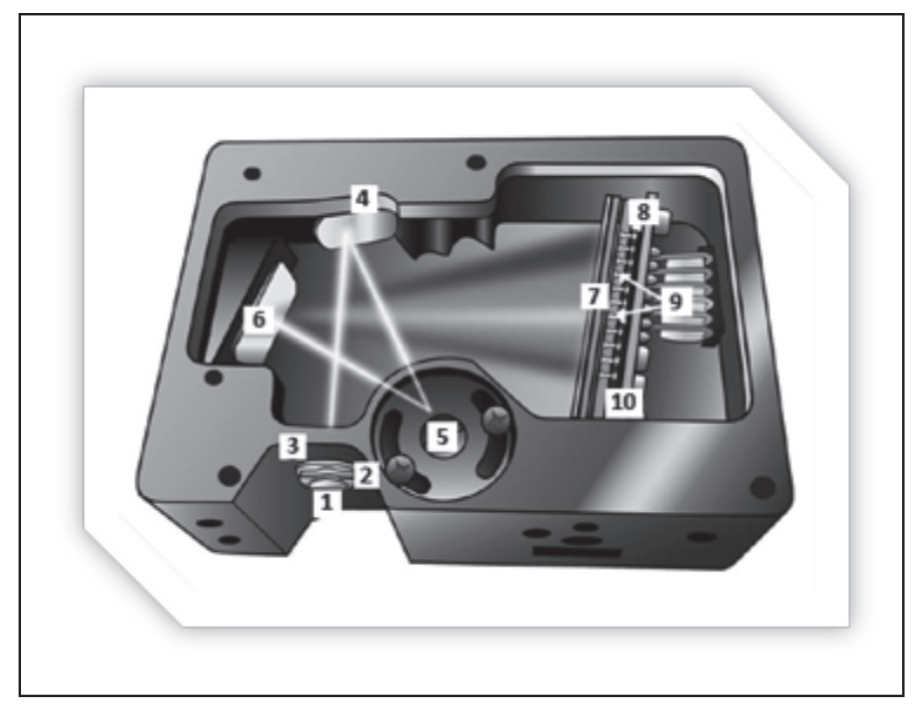

Figura 3. Espectrómetro USB 4000 UV-VIS-ES Ocean Optics [1].

\section{Diseño e implementación del sistema electrónico}

En esta sección se detalla el diseño e implementación del sistema óptico del equipo, que está conformado por la fuente de luz, el detector y la punta de toma de muestras. 


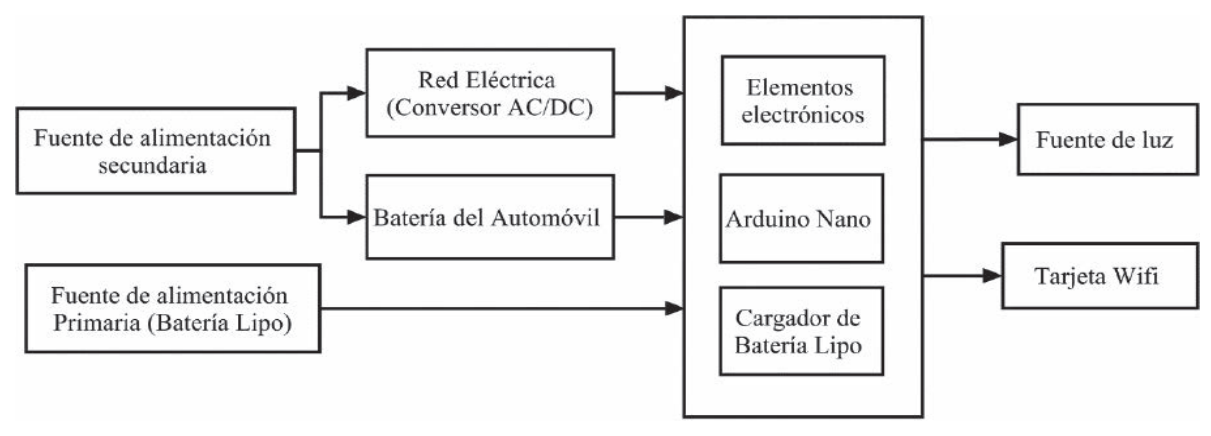

Figura 4. Módulos de la tarjeta electrónica.

\section{d. Diseño de la tarjeta electrónica}

La tarjeta electrónica consta de los siguientes elementos electrónicos:

- Transistor BJT

- Transistor Mosfet

- Relay Dc.

- Regulador de voltaje

- Amplificador operacional

- Resistencias

- Capacitores

- Conectores

La tarjeta centraliza el acople entre los módulos constitutivos del equipo y el sistema de control para ello se ha establecido la utilización de conectores estándar y las dimensiones de cada módulo constitutivo, el diseño también acopla la tarjeta de desarrollo Arduino Nano, mediante esta se ha establecido el sistemas de control [6] que activa el módulo la fuente de luz halógena, la tarjeta de comunicación, el detector USB4000 UV-VIS-ES, el sistema de alarma, sistema de visualización y el sistema de carga de batería [7], mostradas en la figura 5 . 


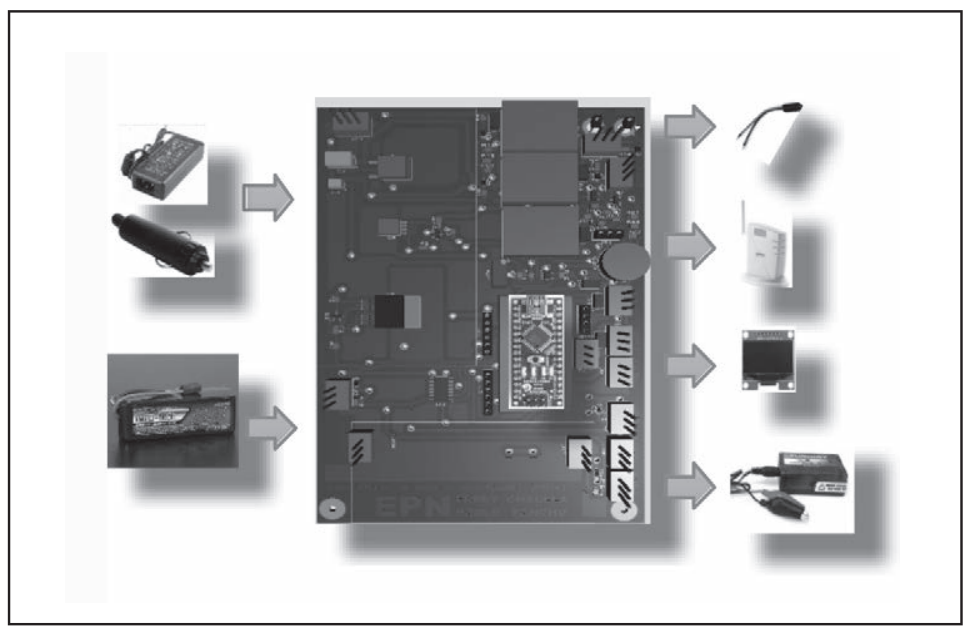

Figura 5. Módulo constitutivo del sistema espectral.

La implementación de un sensor de presencia electromagnética en la punta de toma de muestras permite iniciar la secuencia de control para el encendido de la fuente de luz al momento de tomar la muestra en las hojas de las plantas vegetales, las notificaciones del estado de la batería y alarmas son mostradas en la pantalla Oled en todo momento en el equipo. Para la carga de batería el prototipo dispone de dos puntos de carga mediante la red de $110 \mathrm{Vac}$ o el conector de $12 \mathrm{Vdc}$ del automóvil.

\section{Software de visualización}

En esta sección se describe la elaboración del software de visualización que permitirá graficar los espectros de las muestras tomadas.

\section{e. Desarrollo de la interfaz gráfica de usuario}

Una interfaz gráfica es un vínculo entre un programa computacional y el usuario, constituido generalmente por un conjunto de comandos y menús, por medio de los cuales el usuario se comunica con el programa durante las operaciones que se deben realizar, facilitando la entrada y salida de datos e información.

El GUIDE de Matlab consta de los siguientes archivos:

- Archivo .fig Este archivo contiene los elementos gráficos que son programados mediante código contenido en al archivo .m.

- Archivo .m Este archivo es el que contiene el código de los distintos componentes del GUIDE como son botones, axes, group buttons, etc.

El diseño de la interfaz de control se realiza en la plataforma de desarrollo Matlab [8]. La interfaz consta de los siguientes elementos: 
- Push Button: genera una acción.

- Radio Button: representa una opción.

- Check Box: indica el estado de una opción.

- Edit Text: sirve para editar texto.

- Axes: sirve para graficar.

- Button Group: es un panel que se utiliza solo para radio buttons y toggle buttons. El diseño de la interfaz se lo realiza en la ventana de trabajo GUIDE que se observa en la figura 6 .

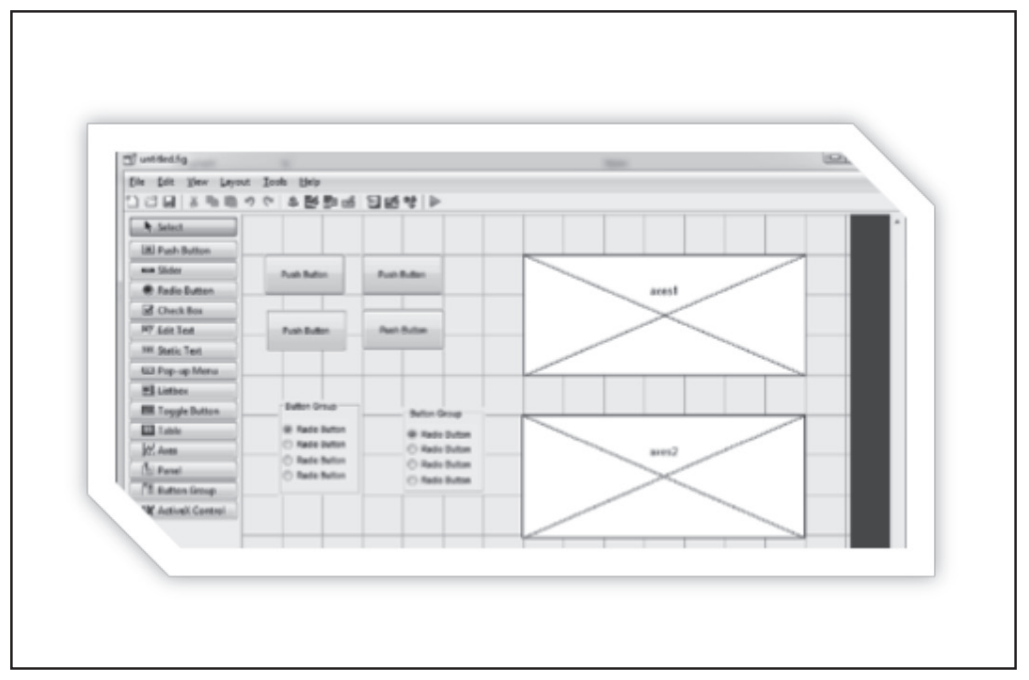

Figura 6. Área de trabajo Blank Guide de Matlab.

En esta área de trabajo se arrastra los elementos que se utilizan y se los ordena de acuerdo al gusto y preferencias del usuario [9]. Después de escoger los elementos gráficos que se mostraran en la pantalla, se debe programar las órdenes que deben cumplir cuando sean manipulados.

Para este proceso se ocupa el otro archivo que proporciona Matlab para la elaboración de la interfaz. Mediante el archivo .m se colocan, empleando código de programación las tareas y acciones que cumple cada componente presente en la interfaz. La interfaz consta de los siguientes componentes con su determinada función y se los puede observar en la figura 7 . 


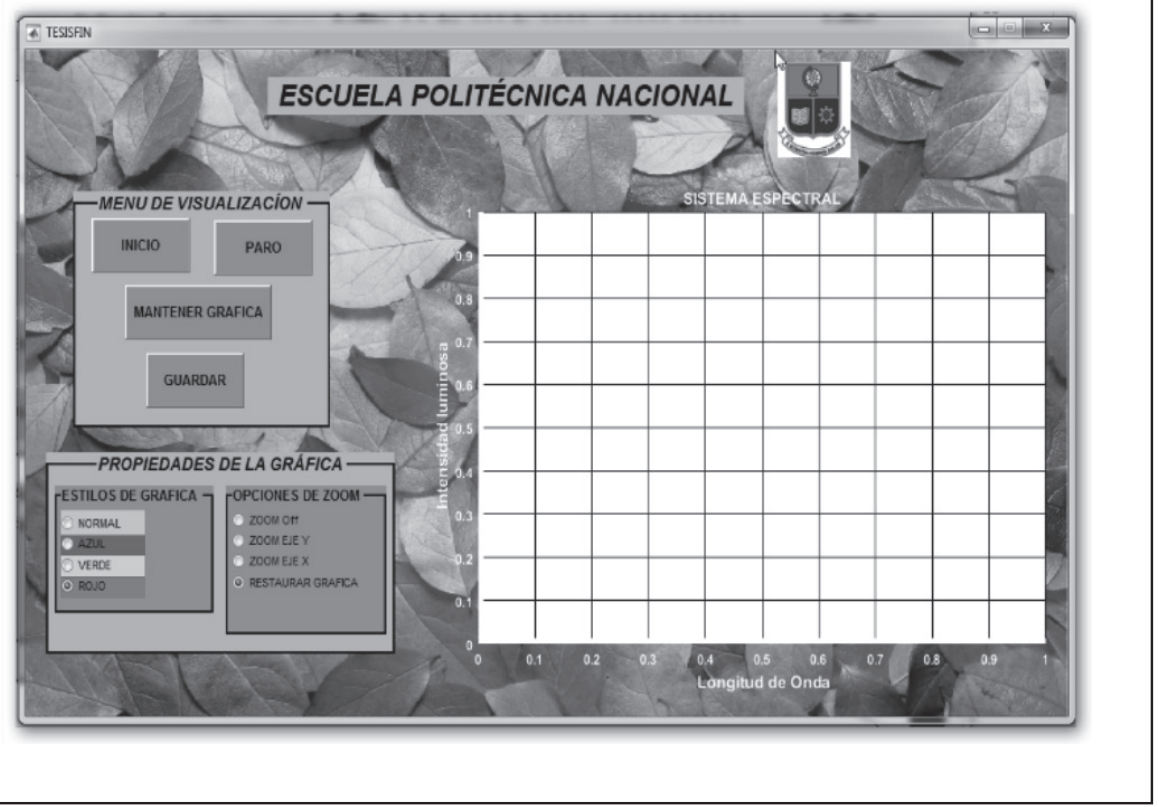

Figura 7. Interfaz gráfica final.

- Inicio

Componente de tipo "Push Button", sirve para que el espectrómetro empiece a tomar datos y a graficarlos. Mediante este botón se obtiene la gráfica del instante en que se lo presiona.

- Paro

Componente de tipo "Push Button", funciona como paro del software, este se encarga de cerrar la conexión del espectrómetro con la computadora.

- Guardar

La función de este botón es guardar la imagen graficada en un archivo jpg, cuando se toma datos de una muestra y se la gráfica, este botón permite guardar la imagen que se desea conservar para posteriores usos.

- Mantener gráfica

Este botón sirve para realizar múltiples gráficas, cuando se quiere realizar una comparación entre dos o más gráficas al mismo tiempo, mediante este botón las gráficas se sobreponen y se puede visualizar más de una a la vez. 
- Estilos de gráfica

Este componente sirve para agrupar botones, los cuales tienen funciones que se relacionan, la función que relaciona a este grupo de componentes son los estilos de la gráfica visualizada.

- Normal

Este componente es parte del "button group" de estilo de gráfica, en donde el estilo de gráfica es normal por defecto del programa, esto quiere decir que se realiza la gráfica en color negro y línea continua.

- Azul

Este componente es parte del button group de estilo de gráfica, en donde el estilo de gráfica se modifica con un cambio de color y línea con la que se dibuja la gráfica, para este estilo se cambia a color azul la gráfica y a línea entrecortada.

- Verde

Este componente es parte del button group de estilo de gráfica, en donde el estilo de gráfica se modifica con un cambio de color y línea con la que se dibuja la gráfica, para este estilo se cambia a color verde la gráfica y a línea entrecortada con puntos.

- Rojo

Este componente es parte del button group de estilo de gráfica, en donde el estilo de gráfica se modifica con un cambio de color y línea con la que se dibuja la gráfica, para este estilo se cambia a color rojo la gráfica y a línea dibujada con asteriscos.

- Opciones de zoom

Este componente sirve para compaginar un grupo de botones los cuales tienen funciones que se relacionan, la función que los relaciona para este grupo de componentes son las opciones de zoom de la gráfica visualizada.

- Zoom off

Este componente es parte del button group de opciones de zoom, este radio button está activado por defecto por lo que el zoom está desactivado.

- Zoom eje $\mathrm{Y}$

Este componente es parte del button group de opciones de zoom, este radio button sirve para realizar un zoom en el eje y de la gráfica.

- Zoom eje X

Este componente es parte del button group de opciones de zoom, este radio button sirve para realizar un zoom en el eje x de la gráfica. 
- Restaurar gráfico

Este componente es parte del button group de opciones de zoom, este radio button sirve para regresar al gráfico a su estado inicial sin zoom.

- Axes 1

Este componente sirve para cargar la imagen de fondo de la aplicación.

- Axes 2

Este componente sirve para graficar los datos enviados por el espectrómetro.

\section{Pruebas y resultados}

En esta parte se describen las distintas pruebas realizadas al equipo, así como los resultados obtenidos de las mismas, aquí se compara el accionar del equipo en contraste con un equipo comercial.

\section{f. Sistema electrónico}

Se procede a verificar el funcionamiento del accionar de la fuente de luz, cargador de batería lipo, conversor AC/DC, conector del automóvil, y visualización de mensajes en la pantalla.

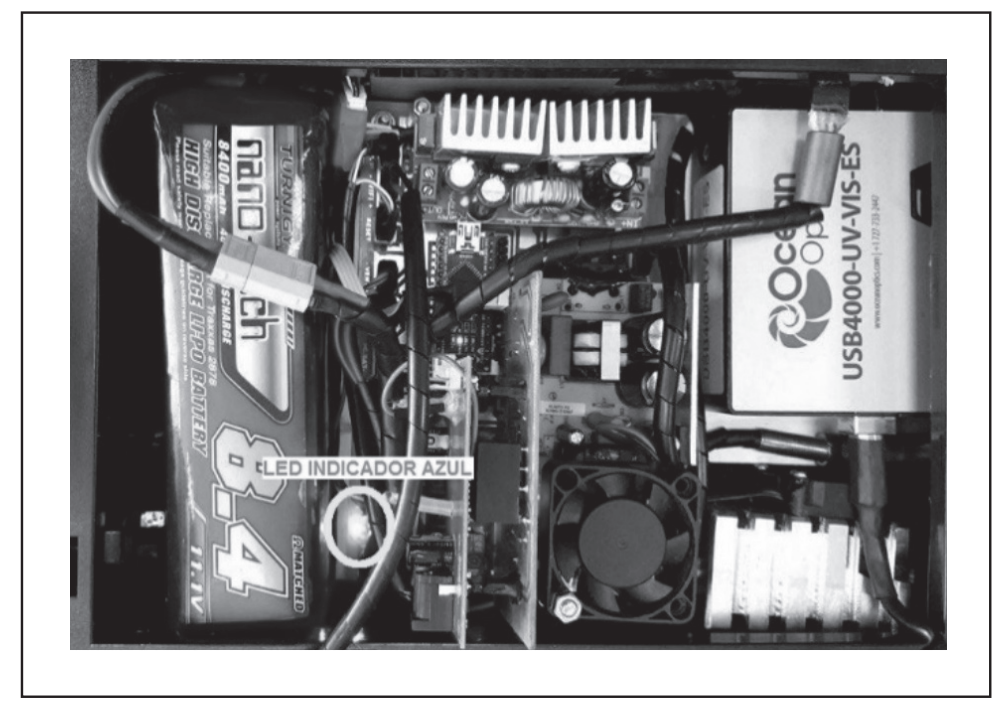

Figura 8. Funcionamiento del equipo mediante la batería lipo.

Luego de accionar el switch que enciende al equipo, se observa que un led de color azul se enciende, esto indica que la alimentación del equipo proviene de la batería lipo. Esta batería se encarga de proporcionar la energía total del equipo, como 
se observa en la figura 8. Cuando el equipo está recibiendo energía de la batería lipo se pueden mostrar tres mensajes dependiendo del nivel de la batería. En la figura 9 se observa el mensaje cuando la batería se encuentra completamente cargada.

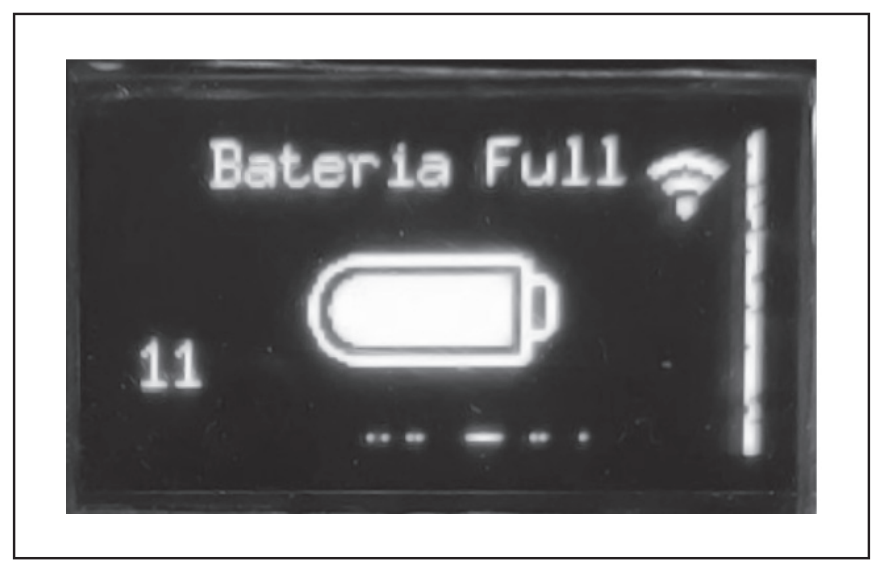

Figura 9. Mensaje de batería full.

En la figura 10 se observa el mensaje cuando la batería está descargada, además se acciona la alarma sonora.

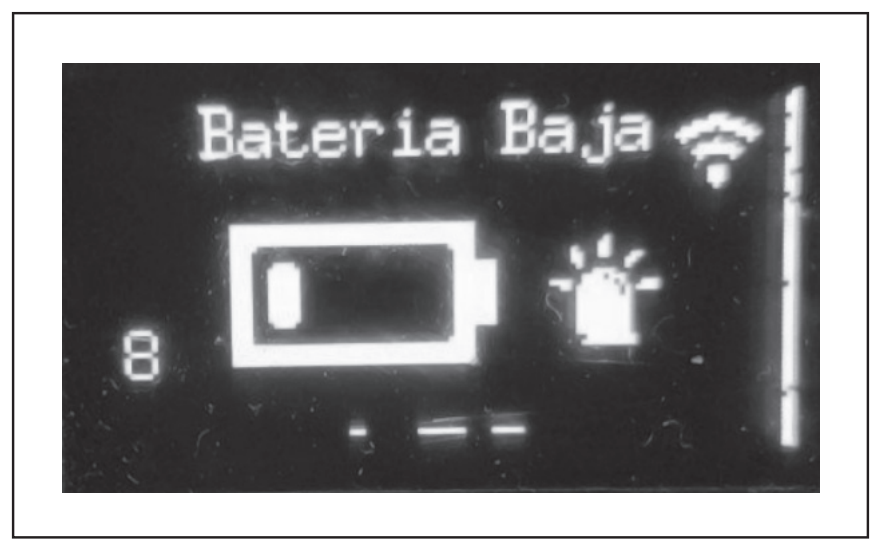

Figura 10. Mensaje de batería baja.

Cuando la batería está descargada se puede conectar tanto a la red eléctrica como al conector estándar del automóvil. Cuando se conecta a la red eléctrica se enciende un led de color verde como se ve en la figura 11. 


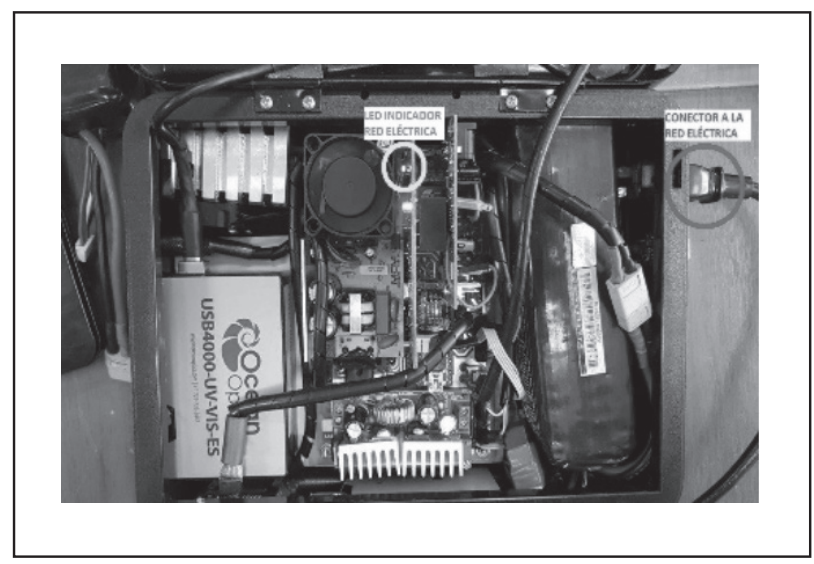

Figura 11. Equipo conectado a la red eléctrica.

Además, en el visualizador se muestra un mensaje referente a esta acción, si la batería está descargada se muestra un mensaje de batería cargando, como se ve en la figura 12 .

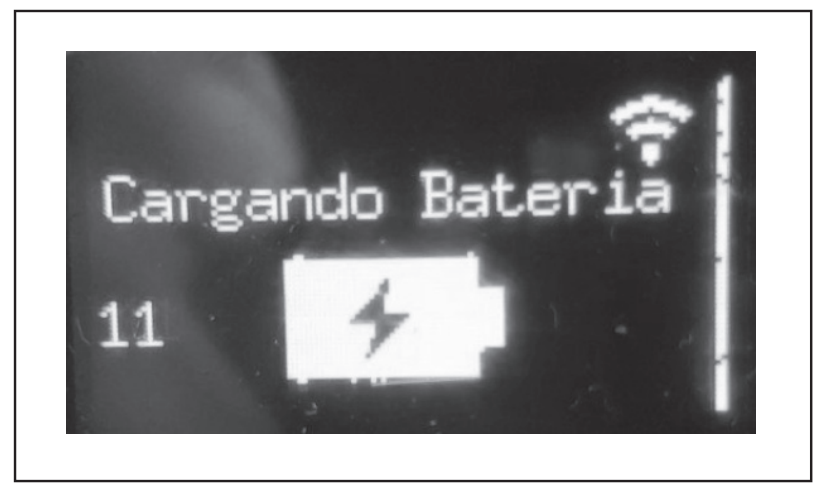

Figura 12. Mensaje de batería cargando.

La segunda opción para proporcionar energía al equipo es mediante un conector estándar de $12 \mathrm{~V}$ presente en los automóviles, en la figura 13, se observa el equipo funcionando mediante este conector, en la tarjeta se enciende un led de color blanco que indica que el equipo se ha conectado al automóvil. 


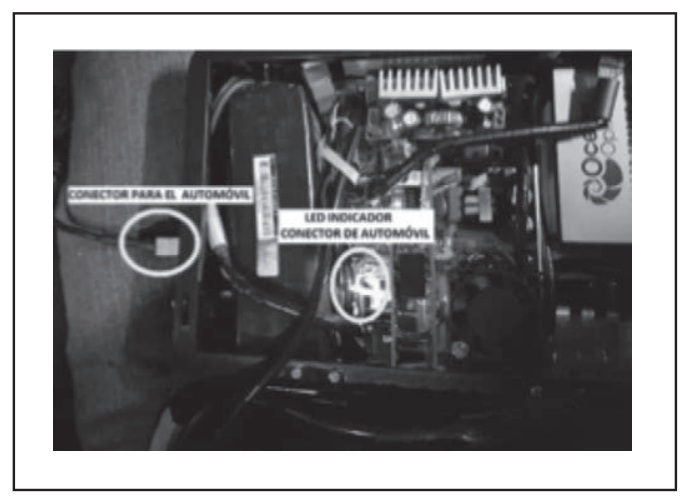

Figura 13. Equipo conectado al automóvil.

La fuente de luz se activa mediante la punta de toma de muestras al cerrarse y se apaga cuando esta se abre, este proceso se observa en las figura 14-15.

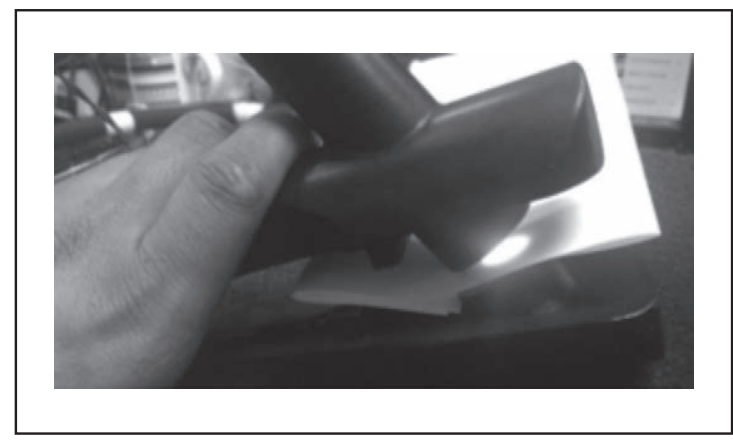

Figura 14. Encendido de la fuente de luz mediante la punta de toma de muestras.

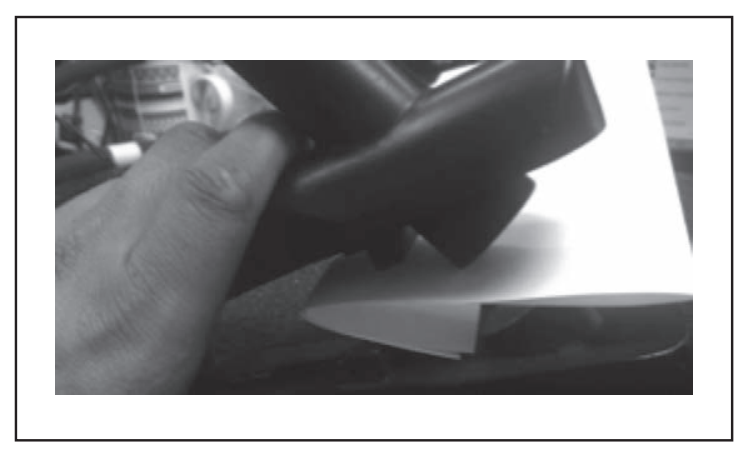

Figura 15. Apagado de la fuente de luz mediante la punta de toma de muestras. 


\section{g. Software}

Para verificar el software se utilizó el láser de 337 nm. En la figura 16 se puede observar el pico que presenta el láser.

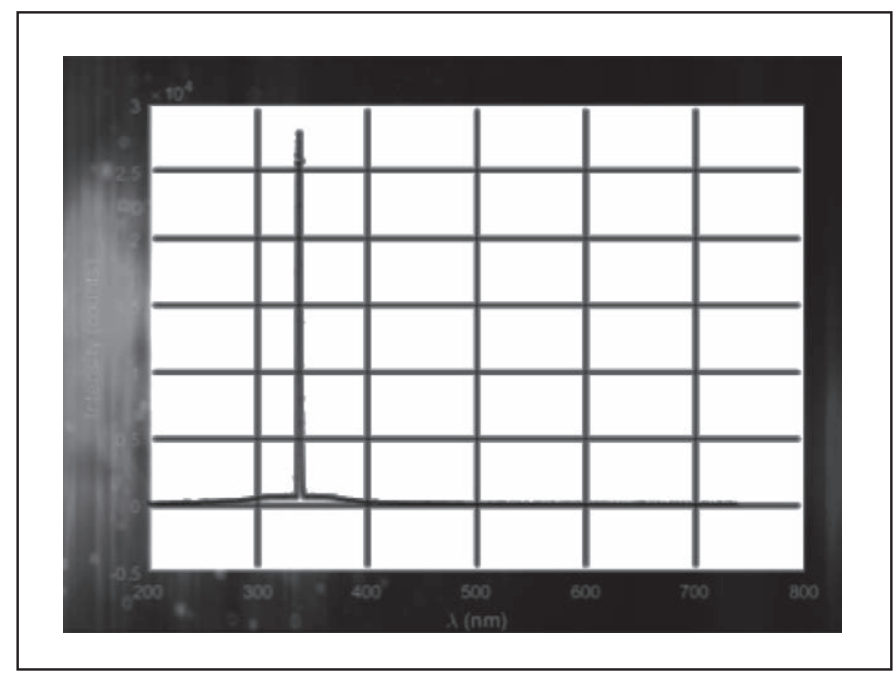

Figura 16. Muestra tomada mediante el espectrómetro USB-4000 UVVIS-ES con el pico del láser UV en 337 nm y el software desarrollado en el proyecto.

Se tomó muestras utilizando el equipo implementado a una hoja de geranio como se muestra a continuación en la figura 17 .

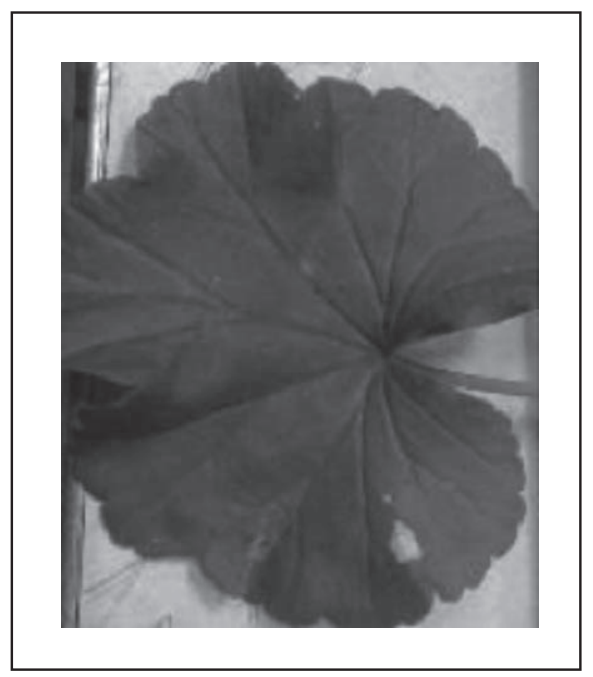

Figura 17. Hoja de geranio. 
La figura 17 muestra una hoja de geranio, de esta hoja se obtuvo el espectro mostrado en la figura 18, para lo cual se estableció el área a ser analizada, luego se obtuvo la primera muestra con el equipo FieldSpec 4, a continuación con el equipo desarrollado. Al momento de analizar los espectros se establece que la forma espectral corresponde al igual que su longitud de onda de eje " $x$ ", con respecto al eje " $y$ " difiere por la intensidad de luz aplicada por cada equipo.

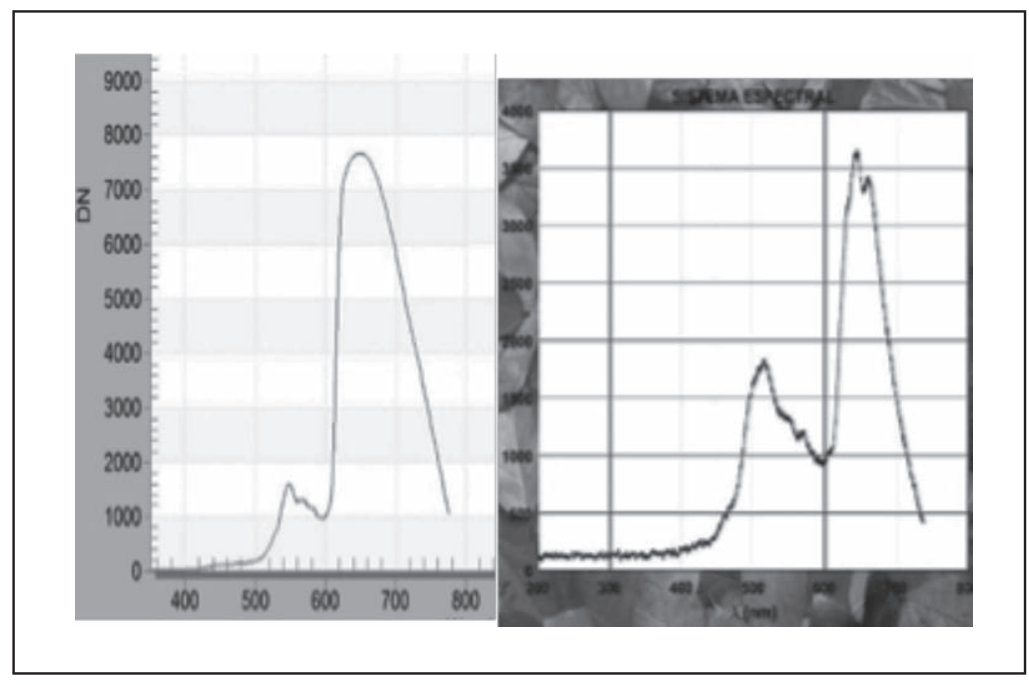

Figura 18. Muestras tomadas con el equipo comercial vs. el equipo implementado a una hoja de geranio.

\section{Conclusiones}

Se logró implementar la tarjeta electrónica de control, permitiendo que el equipo funcione sistemáticamente, gestionando sus elementos de acuerdo al código de programación desarrollado, empezando por fuente de luz, tarjeta de comunicación wifi, detector USB4000 UV-VIS-ES, cargador de batería y visualizador gráfico.

Se desarrolló un software que consta de un menú de opciones permitiendo realizar la toma de datos de manera directa, ayudando al usuario a elegir el tipo de color, opciones de zoom y guardar las gráficas para posteriores análisis.

El visualizador implementado permite que el usuario conozca en todo momento el estado de la batería. Por medio de una alarma sonora el sistema de control advierte cuando el equipo necesita recargar la batería, indicando al usuario que el nivel no es el suficiente para que el equipo siga funcionando. Además, se logró implementar un sistema de carga de batería práctico, que alimenta al equipo y carga la batería mediante la red eléctrica o mediante el automóvil, que es más común en salidas al campo. 
Se logró diseñar e implementar la punta de toma de muestras adecuada al equipo, de modo que se ajusta con facilidad al ambiente de trabajo, por el tamaño y peso, puede acceder a lugares angostos y poco accesibles, lo que permite al usuario obtener muestras sin mayor problema. Además, el sensor magnético implementado en la punta de toma de muestras acciona la fuente de luz, evitando que esté encendida todo el tiempo, optimizando de esta manera su uso.

El tamaño de la tarjeta electrónica diseñada disminuyó gracias al uso de elementos electrónicos de montaje superficial (SMD) y al trazado del circuito a doble lado, optimizando el tamańo que la tarjeta ocupa en el equipo.

El envío de datos se realiza mediante un servidor USB, lo que permite enlazar el equipo con toda computadora que posea tecnología wifi, con un alcance de 100 metros con línea de vista, que permite cubrir más terreno para la toma de muestras.

Las pruebas realizadas muestran que el software desarrollado proporciona gráficas fiables en el rango establecido en el plan de trabajo, además en comparación con un software comercial, cumple con sus objetivos ya que posee las funciones necesarias para visualizar los datos obtenidos, siendo portable y de fácil manipulación.

\section{Recomendaciones}

El desarrollo de la aplicación se recomienda realizarlo en softwares afines con Android, que permite ejecutarlas mediante una tablet o un celular, esto sirve como base para poder realizar un equipo más comercial y que tenga mayores facilidades para una transferencia tecnológica.

Se recomienda realizar un sistema de energía portable, con el cual el equipo reduzca su peso y tamaño, permitiendo al usuario recargar las baterías sin necesidad de movilizar todo el equipo.

Por la sensibilidad que tienen la tarjeta de carga de batería se recomienda utilizar conectores estándares y circuitos de protección para evitar daños en tarjeta.

Para futuros equipos espectrales se debería aplicar un conversor AC/DC diseñado específicamente para el equipo, evitando tamaños excesivos de este módulo, con el fin de reducir más las medidas del prototipo desarrollado.

Una solución para evitar depender de cables soldados a la placa son conectores Molex, mediante estos se acopla y desacopla de forma segura los periféricos externos a la placa electrónica, ofreciendo seguridad en el montaje y desmontaje del equipo.

La ubicación física de la tarjeta Arduino en la placa electrónica es para evitar que el programa de control desarrollado sea cargado de forma directa, esta medida de seguridad evita que el puerto USB del computador se dañe ya que la tarjeta electrónica desarrollada exige más corriente de la que entrega. 


\section{Referencias}

[1] «Ocean Optics», HL-2000 Family, 2017. [En línea]. Available: https://oceanoptics. com/product/hl-2000-family/. [Último acceso: 12 Abril 2017], Insolab, «Fuente de luz de tungsteno halógena Modelo HL-2000», [En línea]. Available: http:// www.insolab.com.co/component/mijoshop/product/165-fuente-de-luz-de-tungsteno-halogena-modelo-hl-2000. [Último acceso: 20 marzo 2017]

[2] H. Zajac, de Óptica, S.A. Apartado Postal 62361 Caracas 106, Venezuela. Apartado Aéreo 29696 Bogotá, Colombia. Apartado Postal 19-188 México 19 D.F, México. Apartado 4289 Panamá 5, Panamá. Fondo Educativo Interamericano, 2010.

[3] O. Optics, «74-series Collimating Lenses», [En línea]. Available: https://oceanoptics.com/product/collimating-lens-74-series/. [Último acceso: 15 marzo 2017].

[4] O. Optics, «Fibers y Probes», [En línea]. Available: info@OceanOptics.com. [Último acceso: 7 marzo 2017].

[5] O. Ocean, «USB4000-UV-VIS», [En línea]. Available: https://oceanoptics. com/product/usb4000-uv-vis-es/. [Último acceso: 9 marzo 2017].

[6] J. C. V. Ferreira, Microcontroladores Motorola - Freescale: programación, familias y sus distintas aplicaciones en las industrias. México: Alfaomega grupo editorial, S.A. de C.V., 2008.

[7] T. Floyd, Dispositivos electrónicos. México: Pearson Education, 2008. FLEXChip Signal Processor (MC68175/D), Motorola, 1996.

[8] Matlab, «MathWorks», 2017. [En línea]. Available: https://es.mathworks.com/ products/matlab/features.html. [Último acceso: 10 abril 2017]. [8] FLEXChip Signal Processor (MC68175/D), Motorola, 1996

[9] L. Llamas, "Ingeniería Informática y Diseño», [En línea]. Available: https:// www.luisllamas.es/arduino-transistor-mosfet/. [Último acceso: 4 abril 2017]. 